\title{
Microstructure Evolutions at Severely-deformed Austenite/Martensite Interfaces of a Layer-integrated Steel
}

\author{
Taisuke HAYASHI, ${ }^{1)}$ Eiji ABE, ${ }^{1)}$ Toru HARA ${ }^{2)}$ and Yuichi IKUHARA ${ }^{3)}$ \\ 1) Department of Materials Engineering, The University of Tokyo, 7-3-1 Hongo, Bunkyo-ku, Tokyo $113-8656$ Japan. \\ E-mail: abe@material.t.u-tokyo.ac.jp $\quad$ 2) National Institute for Materials Science, 1-1 Namiki, Tsukuba, Ibaraki 305-0044 \\ Japan. 3) Institute of Engineering Innovation, The University of Tokyo, 2-11-16 Yayoi, Bunkyo-ku, Tokyo $113-8656$ \\ Japan.
}

(Received on March 9, 2009; accepted on May 25, 2009)

\begin{abstract}
The microstructure evolution at interfaces of a layer-integrated steel sheet artificially constructed by ductile austenitic stainless (SUS304) and high-strength martensitic (SCM415) steel layers, which were bonded through a cold-rolling and a subsequent annealing at $1000^{\circ} \mathrm{C}$, has been investigated using scanning transmission electron microscopy (STEM) combined with energy dispersive X-ray spectroscopy (EDS). We find that a significant microstructural reconstruction around the SUS304/SCM415 interface has been accomplished during a short-time annealing followed by water-quenching; the resultant microstructures are found to consist of recrystallized austenite and lath martensite grains for the SUS304 and SCM415 layers, respectively. Interestingly, the original SUS304/SCM415 interface appears to migrate and extend into the SUS304 side, an occurrence of which can be reasonably explained by the martensitic transformation across the composition-gradient interface during quenching. These microstructural evolutions fairly account for a microscopic mechanism on how hetero-interface bonding can be achieved via simple cold-rolling/annealing procedures.
\end{abstract}

KEY WORDS: steels; interface structure; scanning transmission electron microscopy; phase transformation; severe plastic deformation.

\section{Introduction}

Multi-layered metals artificially constructed by brittle and ductile layers are of interest because of their good fracture properties, unique mechanical behaviors, ${ }^{1-8)}$ and further possibilities to attain functional properties such as corrosion resistance. As a pioneering work, Sherby et al. investigated mechanical behaviors of laminated composites fabricated by a rolling; e.g., the composites constructed by ultrahigh-carbon-steel/mild-steel ${ }^{9)}$ and by ultrahigh-carbonsteel/brass. ${ }^{5,7,8,10,11)}$ They reported that the strength of the laminated composites basically obeys the rule of average, but the rule is hardly applied for their ductility; a tensile elongation of the composites generally becomes less than the average of the components. ${ }^{7)}$ However, one of the interesting findings is that, when each of the layer thickness was decreased to $50 \mu \mathrm{m}$, the laminated composite of high-carbon-steel/brass reveals the significant elongation of $\sim 60 \%$, which is remarkably more ductile than that expected from the rule of average. ${ }^{12)}$ This surely encouraged a further potential of the multi-layered composites, although it is still not fully understood on how such good ductility can be realized. In any cases, for the development of superior multilayered composites, it is nevertheless an important key process to realize the strong bonding at heterointerfaces between the dissimilar materials.
Very recently, Nambu et al. investigated the tensile and peel behaviors of the multi-layered steel constructed by austenitic stainless (SUS304) and martensitic (SCM415) layers, where the former and the latter provides high deformability and high strength of the laminated composite, respectively. ${ }^{13)}$ For fabrications of the SUS304/SCM415 laminate, a cold rolling process was employed rather than a hot rolling process in order to suppress decarburization and resultant softening of the SCM415 martensitic layer. Of course, the bonding strength between the SUS304/SCM415 layers is quite weak at the as-rolled state, and hence the SUS304/SCM415 laminated composite does not reveal any significant ductility and fractures just after the yielding due to interface delaminations. However, the interface strength is found to be significantly improved by just applying a short time annealing around $1000^{\circ} \mathrm{C}$; strength of the SUS304/SCM415 laminate has reached almost the ideal values expected from the rule of average with a sufficient elongation of approximately $\sim 20 \%{ }^{14)}$ This suggests that the strong bonding between the SUS304/SCM415 steels has been achieved through microstructure reconstructions during the annealing at $1000^{\circ} \mathrm{C}$. In the present work, we investigate the microstructure evolutions around the coldrolled SUS304/SCM415 interfaces using scanning transmission electron microscopy (STEM) combined with energy dispersive X-ray spectroscopy (EDS). On the basis of 
observed microstructural features, we will discuss how the strong-bonding between the austenite/martensite heterointerface can be accomplished during the annealing-quenching procedures.

\section{Experimental Procedure}

The multi-layered steel was produced by a cold rolling process with reduction of about $60 \%$ in total thickness, and then annealed at $1000^{\circ} \mathrm{C}$ for $1-10 \mathrm{~min}$ followed by quenching into water. As shown in Fig. 1, the outer two layers $(0.16 \mathrm{~mm}$ in thickness after rolled) are composed of commercial-grade stainless steel SUS304, and the inner layer $(0.33 \mathrm{~mm}$ in thickness after rolled) is composed of commercial-grade martensitic steel SCM415. Compositions of SUS304 and SCM415 are summarized in Table 1. Details of the manufacturing process of the multi-layered steel are described in elsewhere. ${ }^{13)}$ The as-rolled and annealed samples were cut into small pieces, polished by $\mathrm{SiC}$ abrasive paper, and thinned by a Gatan PIPS ion mill or focused ion beam system for transmission electron microscopy (TEM)

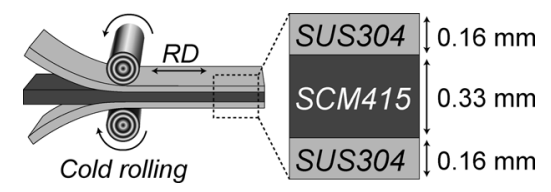

Fig. 1. Schematic illustration of the layer-integrated steel specimen fabricated for the present study.

Table 1. Compositions of the stainless steel SUS304 and martensitic steel SCM415.

\begin{tabular}{lccccccc}
\hline & $\mathrm{C}$ & $\mathrm{Si}$ & $\mathrm{Mn}$ & $\mathrm{Cu}$ & $\mathrm{Ni}$ & $\mathrm{Cr}$ & $\mathrm{Mo}$ \\
\hline SUS304 (wt. \%) & 0.06 & 0.43 & 1.06 & - & 8.04 & 18.18 & - \\
SCM415 (wt. \%) & 0.16 & 0.22 & 0.66 & 0.12 & 0.03 & 1.00 & 0.20 \\
\hline
\end{tabular}

and STEM observations. TEM, STEM, and EDS analysis were performed using a JEOL JEM-2010F field emission gun microscope at acceleration voltage of $200 \mathrm{kV}$. Composition mapping analysis was constructed by using $\mathrm{C}-\mathrm{K}$, $\mathrm{O}-\mathrm{K}, \mathrm{Si}-\mathrm{K}, \mathrm{Mn}-\mathrm{K}, \mathrm{Cr}-\mathrm{K}, \mathrm{Fe}-\mathrm{K}, \mathrm{Ni}-\mathrm{K}$, and $\mathrm{Mo}-\mathrm{L}$ lines.

\section{Results}

\subsection{Microstructure Changes around the Interface}

Figures 2(a)-2(d) show bright-field (BF) STEM images of the SCM415/SUS304 interfaces. For the as-rolled specimen, the microstructures are found to be composed of heavily deformed fine grains both in the SUS304 and SCM415 layers, in which the grains are significantly elongated with large aspect ratios along the roll direction (RD). In particular, the grains in the SUS304 layer, which are composed of deformed austenite and strain induced martensite, exhibit larger aspect ratios because of an intrinsic ductile nature of the austenite grains. These microstructural features indicate that each of the grains has been severely deformed and should contain a considerable amount of resultant strains. After annealing at $1000^{\circ} \mathrm{C}$ for $1-2 \mathrm{~min}$ followed by water-quenching, significant microstructural reconstructions have immediately taken place in both the layers; see Figs. 2(b) and 2(c). Instead of the fine grains with large aspect ratios in Fig. 2(a), the microstructures around the interface fully transformed into those composed of recovered/recrystallized grains with an order of several micrometers, which are identified to be austenite and lath martensite for the SUS304 and SCM415 layers, respectively. It should be noted here that the microstructural reconstruction basically involves the multiple events, as briefly summarized in Fig. 3. Note in particular for the SCM415 layer, the severely-deformed microstructure is firstly fully transformed into $\gamma$ grains during the annealing
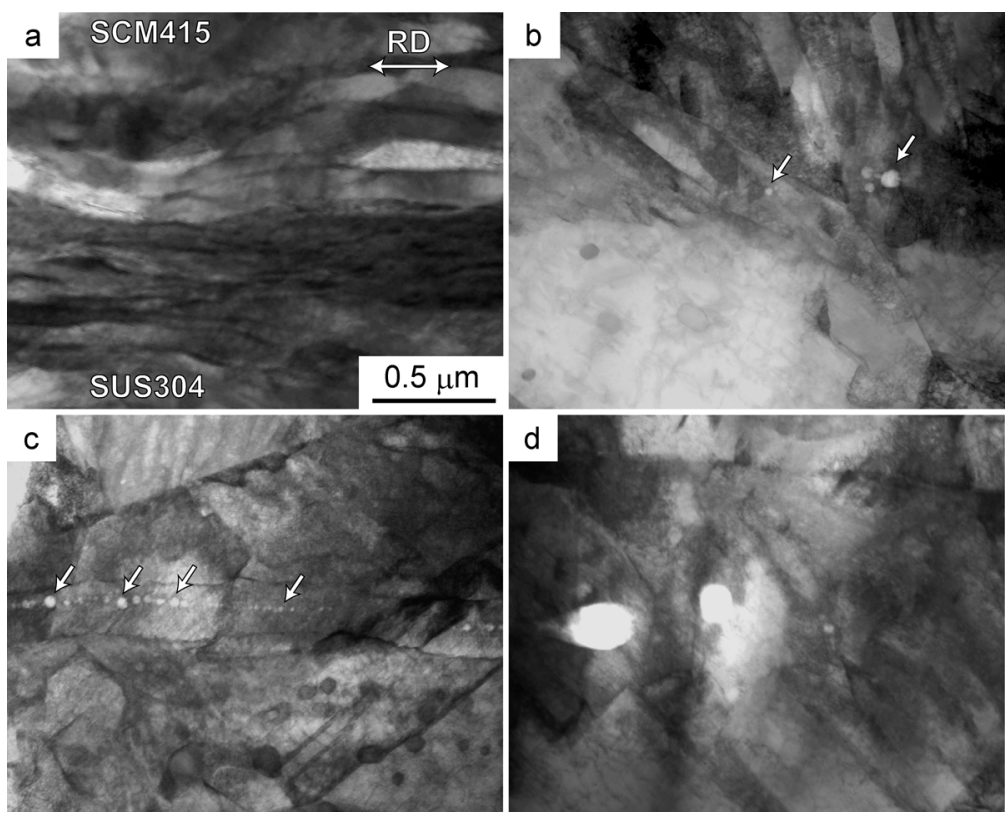

Fig. 2. Bright-filed STEM images obtained from the SCM415/SUS304 interfaces for (a) the as-rolled specimen, and for (b)-(d) the specimens subsequently annealed at $1000^{\circ} \mathrm{C}$ for 1,2 , and $10 \mathrm{~min}$, respectively. Rolling-direction (RD) is parallel to the horizontal line, as indicated in (a).
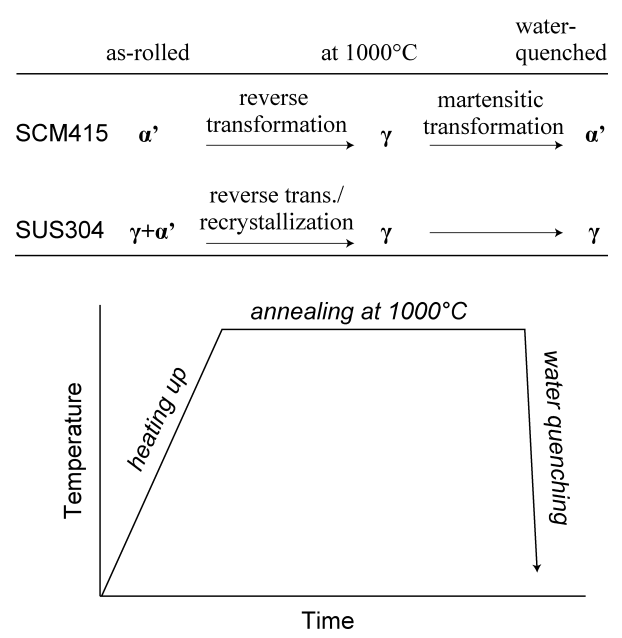

Fig. 3. Schematic diagram of the microstructure/phase change for the SCM415 and SUS304 during the annealing/quenching procedures. 
at $1000^{\circ} \mathrm{C}$, and then the lath martensite grows during the water-quenching. Details of the microstructure formation due to martensitic transformation will be described later.

Remarkable features in Figs. 2(b) and 2(c) are the formation of small particles those appear within the grain interiors, as indicated by arrows. As described later with Figs. 5(b) and 5(c), these particles are mostly identified as a nanocrystalline silicon oxide that is likely to be the most stable oxides in the present alloys according to the Ellingham diagram. Concerning that the interfaces are formed by bonding the free surfaces treated by scratch brushing ${ }^{13)}$ of the SCM415 and SUS304 plates, the oxygen is presumably inherited from a oxygen content at the original surfaces (note that the surface oxide layers remaining even after the scratch brushing can be effectively destroyed during heavy cold-rolling; in fact, complex oxide blocks $(>2-3 \mu \mathrm{m})$ are rarely and sparsely found at the as-rolled interfaces in the low-magnification optical microscope images ${ }^{15)}$ ). It is confirmed that the small oxide particles are always observed around the SCM415/SUS304 interfaces for all the annealed specimens even with the short-time annealing. Therefore, it can be presumed that the silicon-oxide particles have been precipitated at the initial interface in the very early stage of annealing, perhaps being prior to the recovery/recrystallization events around the interface microstructures. With this assumption, the oxide particle alignments can be used as tracers for the initial interfaces, even though these precipitated particles appear to be embedded into the recrystallized grains. This is even seen for the specimen annealed at $1000^{\circ} \mathrm{C}$ sufficiently long time; in Fig. 2(d) the recrystallized-grains grow into an order of $\sim 10 \mu \mathrm{m}$, but the initial interface can be still traced by the oxide particles those appear in the grain interiors. To sum, the sharp SCM415/SUS304 interfaces in the as-rolled specimen becomes somewhat ambiguous due to recrystallization and/or phase transformations during the annealing-quenching procedures, forming the strongly-bonded interface.

\subsection{Chemical Analysis around the Interface}

Local composition analyses by STEM-EDS were performed to reveal precipitation and diffusion behaviors around the interfaces. Figure 4 shows an element map obtained from the as-rolled specimen. In the map, distributions of $\mathrm{Ni}$ and $\mathrm{Cr}$ clearly identify the sharp interface. Even though there exists an original Mn-Mo precipitate in the SCM415 layer (Fig. 4(h)), it is evident from Fig. 4 that significant segregation and/or precipitation around the interfaces did not take place during a severe deformation by the cold rolling. It is noteworthy that, for the as-rolled specimen, no small oxide particles existed at the interface, except for the occasional large oxide blocks $(>\sim 2 \mu \mathrm{m})$ that are simply due to a crush of the original surface oxide. ${ }^{15}$ ) As already described in the previous section, the short-time annealing at $1000^{\circ} \mathrm{C}$ causes a significant microstructural reconstruction around the SCM415/SUS304 interface. Figure 5 shows the representative element map obtained from the 2-min annealed specimen, and we note three characteristic features; i) $\mathrm{Si}-\mathrm{O}$ particles array along the interfaces, ii) interdiffusion of $\mathrm{Ni}$ and $\mathrm{Cr}$ across the interface, and iii) $\mathrm{Cr}-\mathrm{C}$ precipitates within the SUS304 layer.

Firstly, as for the i), the small particles $(10-50 \mathrm{~nm}$ in di- ameters) arrayed along the interface are mostly identified as silicon oxide (Figs. 5(b) and 5(c)), and few particles are attributed to manganese oxide (Fig. 5(h)). As already discussed, these oxide particles were perhaps formed in the very early stage of annealing and hence trace the original as-rolled interface shown in Fig. 4. Here, it is noted again that these particles are already incorporated into the reconstructed grains (Fig. 5(a)) that have grown across the original interface. From the electron diffraction analysis, these grains across the original interfaces are identified as body centered cubic (bcc) structure, being likely to be martensite grains that have been formed during the quenching from $1000^{\circ} \mathrm{C}$ (see Fig. 3). Therefore, it is reasonable to consider that martensite growth started from the SCM415 layer strides over the original interface, forming a new austenite/martensite interface that has extended into the original SUS304 layer. Further details of the microstructure evolutions due to martensitic transformation will be again discussed later.

Second point ii) is that diffusions of $\mathrm{Ni}$ and $\mathrm{Cr}$ from SUS304 to SCM415 indeed took place during the short time annealing, and consequently a certain composition gradient was formed across the reconstructed interface; see Figs. 5(f) and 5(g). By comparison with the corresponding BF-STEM image of Fig. 5(a), the composition gradient continuously occurs within the reconstructed grain interiors. It should be noted that microstructural reconstruction and diffusion events might have proceeded quickly under far from equilibrium conditions during annealing at $1000^{\circ} \mathrm{C}$, since there must exist a considerable amount of strains being stored around the original severely-deformed interface (Fig. 2(a)). Even with this complicated conditions, the sequence of the events during holding at $1000^{\circ} \mathrm{C}$ can be presumed from the observed microstructural features. Firstly, the reconstruction events proceeded very fast with the aid of a significant strain field, which involves recrystallization and $\alpha^{\prime}-\gamma$ reverse transformation. Note that both the SUS304 and SCM415 are within the $\gamma$ single phase field at $1000^{\circ} \mathrm{C}$; the original interface is fully replaced by the $\gamma$ grains whatever their origin, recrystallization or reverse transformation. Then, diffusion of $\mathrm{Ni}$ and $\mathrm{Cr}$ from the SUS304 side took place within these reconstructed $\gamma$ grain interiors. The resultant chemical gradient will remain even when the $\gamma$ grains transforms again into $\alpha^{\prime}$ during quenching, since the martensitic shear-transformation holds a lattice coincidence during the transformation. With this in mind, weak distributions of $\mathrm{Ni}$ and $\mathrm{Cr}$ along the grain boundary in the SCM415 layer, seen in Figs. 5(f) and 5(g), is reasonably attributed to a trace of the diffusion event that has occurred along the $\gamma$ grain boundary during holding at $1000^{\circ} \mathrm{C}$ (note again that SCM415 is fully transformed into austenite at this temperature). This is supported by the fact that $\mathrm{Ni}$ and $\mathrm{Cr}$ were reported to diffuse preferentially along grain boundary for a clad of low alloyed steel and Ni-base austenitic alloys. ${ }^{16,17)}$ Some boundaries without Ni/Cr traces in the SCM415 layer (Fig. 5(a)) correspond to the lath-plate or lath-packet boundaries that have been formed by the martensitic transformation during quenching from $1000^{\circ} \mathrm{C}$.

Thirdly, as mentioned in iii), chromium-carbide particles of $10-200 \mathrm{~nm}$ in diameter are densely precipitated in the SUS304 layer, which were identified as a $\mathrm{M}_{23} \mathrm{C}_{6}$-type car- 


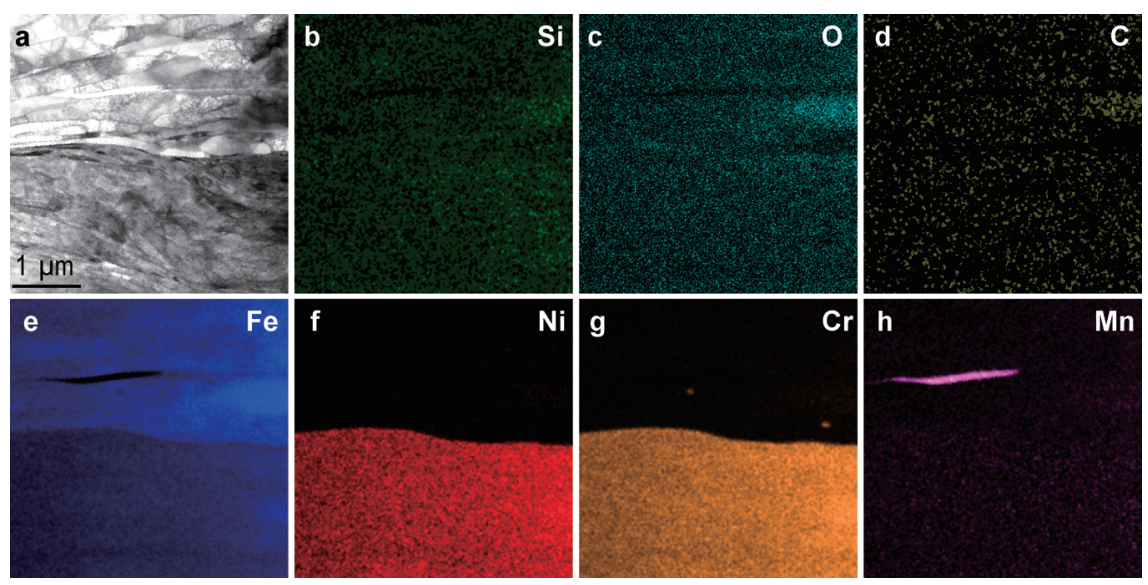

Fig. 4. Element maps obtained from the asrolled SCM415/SUS304 interface: (a) Bright-filed STEM image and the corresponding element distributions of (b)-(h) Si, O, C, Fe, Ni, Cr, and Mn, respectively.

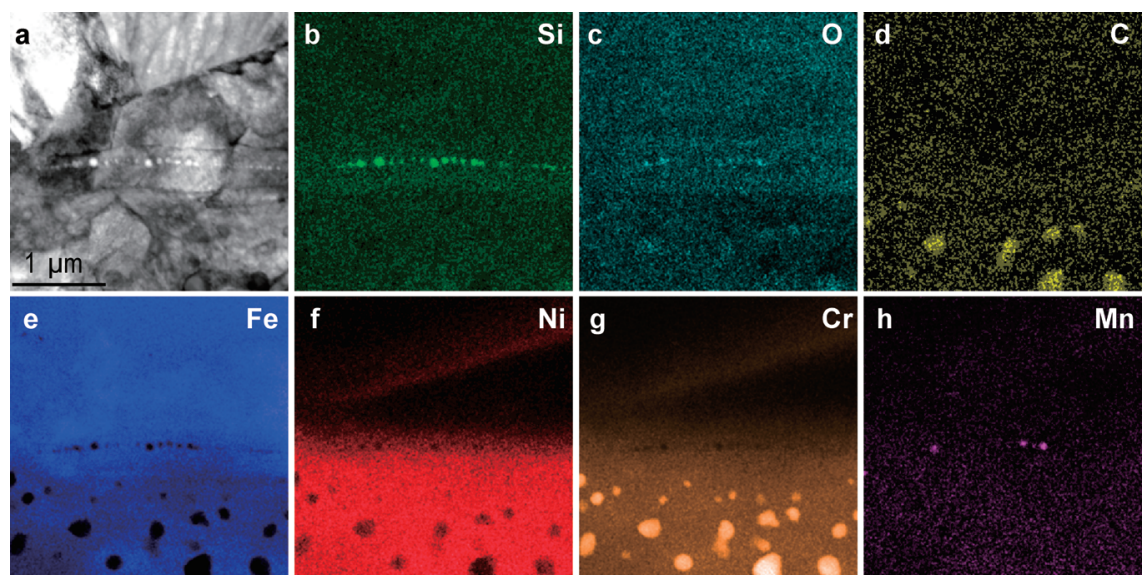

Fig. 5. Element maps obtained from the 2-min annealed SCM415/SUS304 interface: (a) Bright-filed STEM image and the corresponding element distributions of (b)-(h) Si, O, C, Fe, Ni, Cr, and Mn, respectively.
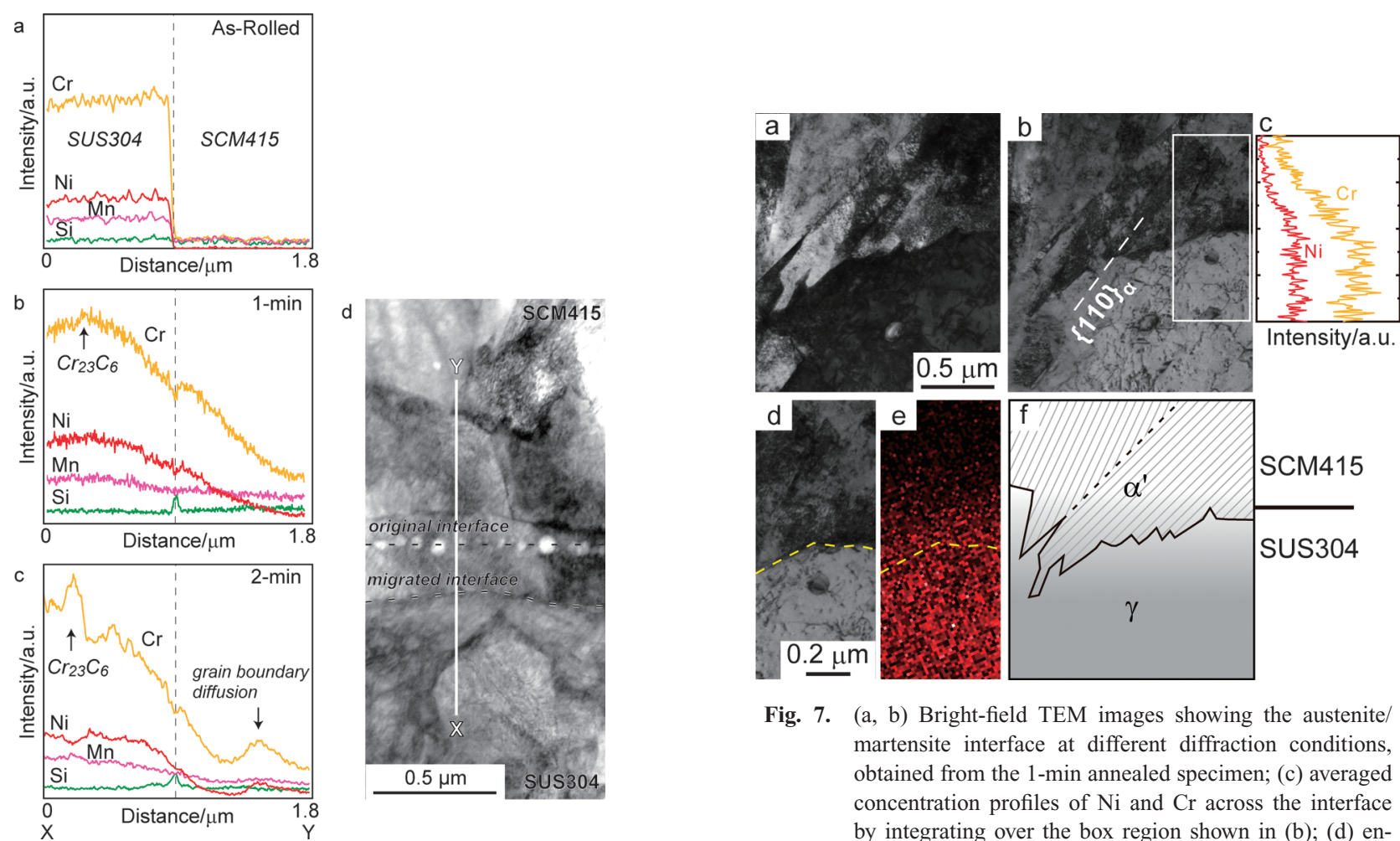

Fig. 7. (a, b) Bright-field TEM images showing the austenite/ martensite interface at different diffraction conditions, obtained from the 1-min annealed specimen; (c) averaged concentration profiles of $\mathrm{Ni}$ and $\mathrm{Cr}$ across the interface by integrating over the box region shown in (b); (d) enlargement of the box region in (b) and (e) the corresponding Ni distribution map; (f) schematic illustration of the martensite-lath morphology and the composition gradient. The dashed line in (f) represents the $\alpha^{\prime}$ packet boundary. as-rolled, (b) 1-min annealed, and (c) 2-min annealed specimens. These profiles are obtained by integrating the spectra over the $\sim 1 \mu \mathrm{m}$ along and the $\sim 2 \mu \mathrm{m}$ across the interface; e.g., the averaged profiles of (b) is obtained from the region of (d), which is an enlarged bright-field STEM image of a part of Fig. 5(a). 
bide by electron diffraction and EDS composition analyses. It was found that these carbides occur only at the band region limited up to $\sim 2 \mu \mathrm{m}$ apart from the initial interfaces (no significant carbides were observed below the region shown in Figs. 5(c) and 5(g)). Dense formation of the carbides in the SUS304 layer indicates that there has been a sufficient carbon supply from the SCM415 into the corresponding band region in the SUS304 layer. This is possible even during the short-time annealing, since the carbon diffusion is generally known to be fast in steels, which could be faster in the present specimen with strongly strained grains. It is noteworthy that there indeed exists a size distribution of the carbide particles depending on the distance from the original interface; they tend to become larger gradually from the near-interface to the far-interface regions. Details of carbide formation around the interface, which should be affected by $\mathrm{Cr}$ diffusion, will be systematically discussed.

Evolutions of element redistributions around the SCM415/SUS304 interface are summarized by the averaged line profiles in Fig. 6. The chemically sharp interface in the as-rolled specimen (Fig. 6(a)) immediately forms composition gradations across the interface during the short time annealing, as shown in Figs. 6(b) (1-min) and 6(c) (2$\min )$. We note that the Si oxide particles already appear for the 1-min annealed specimen, as recognized by the small Si peak in Fig. 6(b). Formation of the $\mathrm{Cr}$ carbide particles was also confirmed for the 1-min annealed specimen, but they were rather less dense compared to those observed for the 2-min annealed specimen. Concentration profiles of $\mathrm{Ni}$ and $\mathrm{Cr}$ in Figs. 6(b) and 6(c) basically reveal a monotonic decrease from the SUS304 to the SCM415 with their slope center almost at the initial interface, except for the local maximum peaks that represent $\mathrm{Cr}$ carbides and a trace of grain boundary diffusion. This suggests that the solute transports of the heavy constituent atoms $(\mathrm{Ni}, \mathrm{Cr}$, etc.) from the SUS304 to SCM415 layers have been achieved mostly through a bulk interdiffusion during annealing. The composition gradient of $\mathrm{Ni}$ and $\mathrm{Cr}$ becomes much broader for the specimens annealed longer than $2 \mathrm{~min}$, and the interface microstructure appear to be complicated with protuberant lath-plates. This will be described later with Fig. 11, in terms of characteristics of the present martensite transformation.

\section{Lath Morphology at the Reconstructed Interface}

During the short time annealing and the following quenching, the original cold-rolled interface is firstly fully covered by the $\gamma$ grains due to recrystallization and $\alpha^{\prime}-\gamma$ reverse transformation at the annealing temperature, and then the martensite laths grow and stride over the original interface during the quenching. Figures 7(a) and 7(b) show the BF images obtained under different imaging conditions, revealing clearly a detailed morphology of the reconstructed interface. From the SCM415 layer, martensite laths are grown into the SUS304 layer with a habit plane parallel to $\{110\} \alpha^{\prime}$. Electron diffraction analysis confirmed that there exists the Kurdjumov-Sachs orientation relationship between the austenite grain and martensite laths shown in Fig. 7. The austenite/martensite boundaries form an overall rectilinear shape, accompanying a nanometer-scaled "ser- rated" morphology at the interface; see the schematic drawing of Fig. 7(f). Occurrence of such serration is simply due to penetration of the martensite laths that are protruded along $\langle 110\rangle \gamma$ with different penetration length. Here, it is noted in particular that the growth of the lath, including the fine laths at the serrated interface, is terminated within the $\gamma$ grain interiors, indicating that the martensite transformation has been somehow terminated within the grain interiors. This is an interesting aspect since the martensite lath generally runs through the $\gamma$ grain entirely from its edge to edge due to a shear transformation nature. With this in mind, we compare the lath morphology with the local compositions; see Figs. 7(c)-7(e). From the comparison between Figs. $7(\mathrm{~d})$ and $7(\mathrm{e})$, the austenite/martensite boundary indicated by dashed lines is found to be definitely located at the region containing a certain amount of $\mathrm{Ni}$. This is further confirmed by the line profiles across the interface (Fig. 7(c)), where the boundary position is at the slope of the $\mathrm{Ni} / \mathrm{Cr}$ concentration profiles. According to this fact, the microstructure evolutions around the interfaces can be speculated as follows. As schematically drawn in Fig. 7(f), the martensite laths were growing from the SCM415 into the SUS304 layer during quenching from $1000^{\circ} \mathrm{C}$, but the growth was interrupted by an existence of the composition gradient, since a driving force of the martensitic transformation decreases with increasing alloying elements including $\mathrm{Ni}$ and $\mathrm{Cr}$. Further details of this consideration will be systematically discussed in the next.

\section{Discussion}

\subsection{Carbide Formation and Element Diffusion Behav- iors Across the Interface}

As described with Figs. 5 and 6, for the SUS304 layer the carbide particles densely exist at the band region laid between $0-2 \mu \mathrm{m}$ apart from the initial interface, and the particles reveal characteristic size distributions depending on the distance from the interface. Similar features of carbide formation were also reported for the weld interfaces between martensitic-steel/stainless-steel or martensiticsteel/Ni-based super-alloy. ${ }^{16,17)}$ Concerning these previous results, the carbide formation process in the present specimen can be presumed as in the followings. At the first step, probably during heating up to $1000^{\circ} \mathrm{C}$, there occurred a sudden change in the $\mathrm{C}$ concentration profile across the ini-

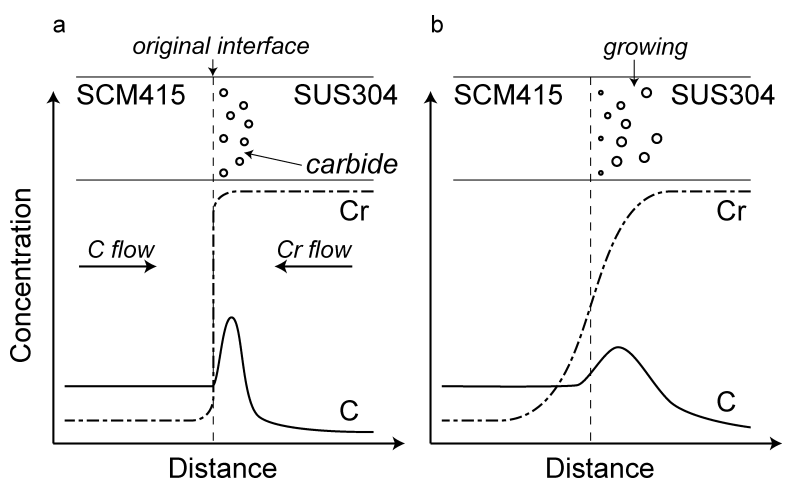

Fig. 8. Schematic illustrations of the carbide formation and the corresponding $\mathrm{Cr} / \mathrm{C}$ concentration profiles at the (a) early stage and (b) later stage of the annealing. 


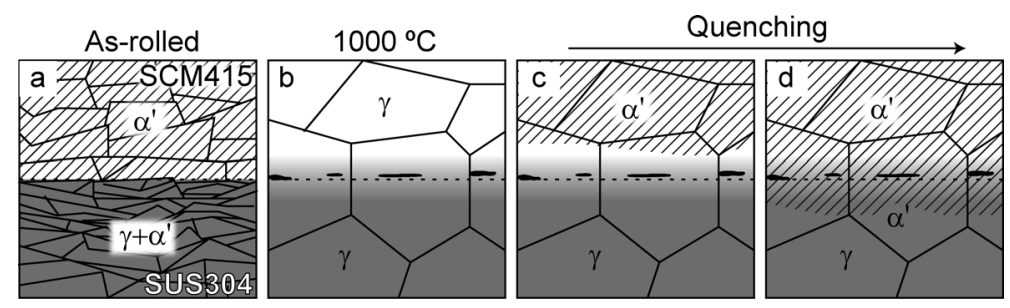

Fig. 9. Schematic illustration of the microstructure evolutions around the SCM415/SUS304 interface. Grey to white gradient schematically represents the compositional gradient between the dissimilar steels.

tial interface at which the $\mathrm{Ni}$ and $\mathrm{Cr}$ concentration is still very steep (Fig. 8(a)), since only the $\mathrm{C}$ atoms are able to move at this situation. This is a reasonable assumption because, in the austenite steels, the diffusion constant of $\mathrm{C}$ is approximately an order of $\sim 10^{-11} \mathrm{~m}^{2} / \mathrm{s}$ at $1000^{\circ} \mathrm{C},{ }^{18)}$ which is much faster than that of $\mathrm{Ni}$ or $\mathrm{Cr}$ interdiffusion in iron matrix with an order of $\sim 10^{-16}-10^{-18} \mathrm{~m}^{2} / \mathrm{s}^{19)}$ Therefore, there would form a sharp peak of C profile in the SUS304 layer next to the initial interface, as schematically drawn in Fig. 8(a), a feature of which was indeed observed for weld joints of ferritic-steel/austenitic-steel. ${ }^{20,21)}$ Since there are sufficient amount of $\mathrm{Cr}$ in the SUS304 layer, the $\mathrm{Cr}_{23} \mathrm{C}_{6}$ type carbide particles immediately precipitate at the occasional C-supersaturated area with a high density of nucleation, forming the carbide band region in the SUS304 layer (Fig. 8(a)). At the second step, when the specimen is almost reached and hold at the annealing temperature $1000^{\circ} \mathrm{C}$, there started extensive interdiffusion of $\mathrm{Cr}$ and $\mathrm{Ni}$, which form an extended concentration gradient across the interface (Fig. 8(b)). During this period, the C profile also developed into a more moderate shape. Given with these concentration profiles shown in Fig. 8(b), the local concentrations of $\mathrm{Cr}$ and $\mathrm{C}$ become higher at the region slightly apart from the interface, whereas the concentrations just near the interface decrease compared to the very early stage of Fig. 8(a). Consequently, the carbides continue growing at the region relatively far form the interface, forming the characteristic size distribution of the carbides as illustrated in Fig. 8(b), which is comparable with Figs. 5(d) and 5(g). It should be noted that the carbide particles nucleated at the near-interface (Fig. 8(a)) could be partially vanished and dissolved into the austenite matrix again at the stage of Fig. 8(b), since a solubility limit of $\mathrm{C}$ in the austenite increases with decreasing the $\mathrm{Cr}$ concentration at this region. This may explain why there are no obvious chromium-carbide particles just below the interface in Fig. $5(\mathrm{~g})$.

Here, it is important to mention that these carbide band regions do not cause significant fracture of the laminated composites, since the present SCM415/SUS304 layer-composite indeed revealed a sufficient elongation of approximately $\sim 20 \%$, where the initial fracture seemed to occur not at the bonded interface. ${ }^{14)}$ Although a further study is necessary, it may be presumed that the present carbides are perhaps still too small to cause significant crack initiations at the carbide/austenite interface.

\subsection{Martensitic Transformation and Strengthening of the Interfaces}

We here discuss how the strong bonding between the dissimilar steels has been achieved through the microstructure evolutions around the interface. As described, the severelydeformed microstructure of the cold-rolled interface has been immediately fully reconstructed during the short-time annealing and the following quenching procedures, which, in fact, remarkably improves the mechanical properties of the SCM415/SUS304 layer-integrated steel. ${ }^{13)}$ This strongly suggests that the reconstructed microstructure itself is responsible for the strong bonding at the SCM415/SUS304 interface. On the basis of the observed microstructural features described in the previous sections, the microstructure evolution around the austenite/martensite interface is summarized as schematically drawn in Fig. 9. The severely-deformed microstructures (Fig. 9(a)) are immediately recrystallized/transformed into that composed of the $\gamma$ grains when the specimen is reached and hold at $1000^{\circ} \mathrm{C}$ (Fig. 9(b)). During holding at $1000^{\circ} \mathrm{C}$, significant growth of the $\gamma$ grains takes place continuously to stride across the initial interface, and then, the long-range diffusion of heavy constituent atoms (Ni, Cr, etc.) across the SCM415/SUS304 interface occurs to form composition gradient within the grain interiors, as schematically drawn in Fig. 9(b). At this moment, it is not clear whether the origin of the $\gamma$ grains those cover the initial interface is due to recrystallization from the SUS304 layer or $\alpha^{\prime}-\gamma$ reverse transformation from the SCM415 layer. In any case, we presume that the "bonding" between the dissimilar steels is primary accomplished at this stage, since the original heterointerface structure is fully replaced by the recovered/recrystallized austenite grains with a moderate composition gradient across the original interface.

Even though the strong bonding is almost obtained at the annealing stage of Fig. 9(b), the SCM415/SUS304 layercomposite is necessary to be quenched to form again the hard martensite structure for the SCM415 layer. During quenching from $1000^{\circ} \mathrm{C}$, martensitic transformation starts from the SCM415 layer and grow towards the SUS304 side (Fig. 9(c)), and the growth of the lath martensite is interrupted by the composition gradient that exists within the grain interiors (Fig. 9(d)). It should be noted that the martensite laths are terminated at the area where the $\mathrm{Cr}$ and $\mathrm{Ni}$ concentrations are slightly lower than those of the SUS304, as described in Fig. 7. With this point of view, the local $\mathrm{Ni} / \mathrm{Cr}$ compositions of the lath-terminated point seem to be well consistent with the Schaeffler diagram, ${ }^{22)}$ in the sense that the local composition values (rough evaluations give $\sim 13 \pm 2 \mathrm{wt} \% \mathrm{Cr}$ and $\sim 7 \pm 2 \mathrm{wt} \% \mathrm{Ni}$, corresponding to $\sim 14 \pm 2$ Cr-equivalent and $\sim 10 \pm 2 \mathrm{Ni}$-equivalent in the diagram) would fall into the austenite/martensite coexisting region in the diagram. From these facts, it can be concluded that the termination of the martensite-lath growth within 
the grain interiors is due to an insufficient driving force of the martensite transformation. That is, when the laths are growing along the composition gradient between the SCM415 and SUS304, the driving force of the martensitic transformation gradually decreases with increasing $\mathrm{Ni}$ equivalent. Figure 10 shows a schematic illustration of a gradual change of the local driving force $\Delta G^{\gamma \rightarrow \alpha^{\prime}}$ along the composition gradient interface, explaining qualitatively that the martensite transformation can not proceed any longer at the position where the sufficient $\Delta G^{\gamma \rightarrow \alpha^{\prime}}$ is not given. This composition-dependent martensite behavior ${ }^{23)}$ can be further confirmed for the SCM415/SUS304 specimen annealed for a longer time, by which more extensive interdiffusion across the interface is expected. Figure 11 shows the representative lath features observed for the specimen annealed at $1000^{\circ} \mathrm{C}$ for $10 \mathrm{~min}$, together with the concentration profiles of $\mathrm{Ni}$ and $\mathrm{Cr}$ measured by EDS. In the image of Fig. 11(a), a few martensite laths are significantly protruded but still terminated within the austenite grain interiors, similar to those observed for the short-time annealed specimens. By comparison with the concentration profiles of Fig. 11(b), where the slopes become more gentle and extended than those of the short-time (1-2 min) annealed specimens, the termination points are still found to be located at the $\mathrm{Ni} / \mathrm{Cr}$ compositions slightly less than those of

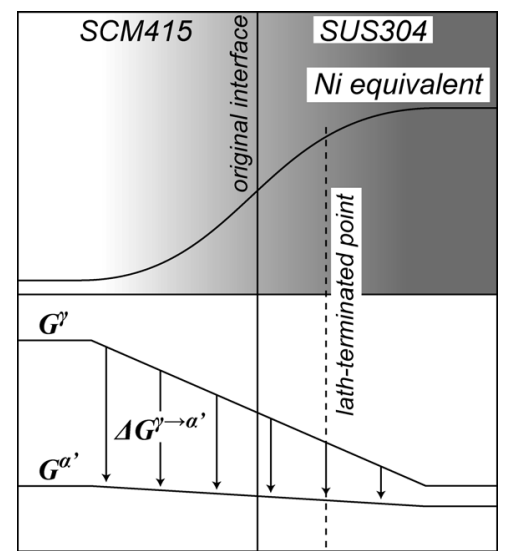

Fig. 10. Schematic diagram of the Ni-equivalent profile and the change of local mean driving force for the martensitic transformation across the interface. the SUS304. Again, the Ni/Cr-equivalent values seem to be consistent with those expected from the Schaeffler diagram.

It is worth mentioning that there may occur a certain amount of strain localized around the lath tip in the austenite grain interiors, because the martensitic shear-transformation involves bulk expansion and resultant strain that accumulates to the lath tips and causes large strain to the adjacent austenite host lattice. One intuitively imagines that these localized strain near the bonding interface may seriously cause crack initiation points; though, it is reminded again that the present SCM415/SUS304 layer-composite indeed revealed a sufficient elongation, and the initial fracture did not occur at the bonded interface. ${ }^{13)}$ We note that, for the present case, fortunately the austenite host lattices are in the fully recrystallized grains, and the transformationinduced strain accumulated at the lath tip may rather easily be relaxed by the sufficiently "soft" austenite matrix, through a mechanism like an effective emission of dislocations from the lath tips. ${ }^{24)}$ Accordingly, the localized strain will effectively be released from the martensite lath tips, preventing crack initiation at the tips located adjacent to the SCM415/SUS304 interface. All in all, the evolution of the martensite microstructures across the interface does not weaken but efficiently achieves the strong bonding of the present layer-composites, which is constructed by hard martensite (SCM415) and soft austenite (SUS304) steels.

\section{Summary}

The microstructure evolutions around the cold-rolled SCM415/SUS304 interface, which have proceeded during the short-time annealing and the following water-quenching procedures, have been investigated using STEM-EDS in order to clarify a microscopic mechanism on how heterointerface bonding can be achieved through the microstructural reconstructions. Conclusions of the present work are briefly summarized in the followings.

(1) STEM-EDS analysis around the SCM415/SUS304 interface has clearly identified the local compositions at submicron scales; formation of silicon-oxide particles $(2-20 \mathrm{~nm})$ and chromium-carbide particles $(2-200 \mathrm{~nm})$, and the moderate $\mathrm{Ni} / \mathrm{Cr}$ composition gradient developed along a few micrometers across the interface. It is presumed that
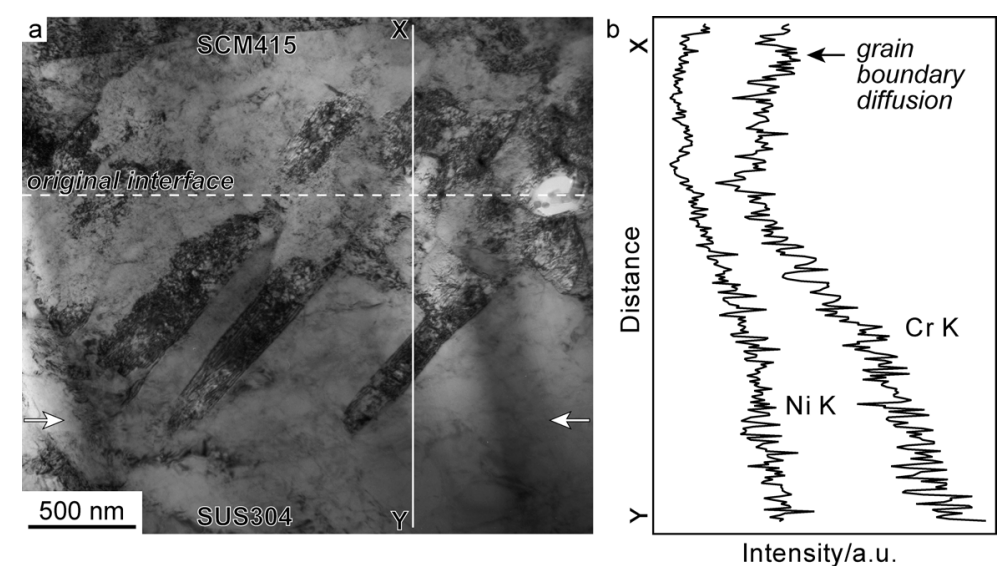

Fig. 11. (a) Bright-field TEM image of the SCM415/SUS304 interface obtained from the specimen annealed at $1000^{\circ} \mathrm{C}$ for $10 \mathrm{~min}$ followed by water-quenching; (b) averaged $\mathrm{Cr} / \mathrm{Ni}$ concentration profiles across the interface, along $\mathrm{X}-\mathrm{Y}$ indicated in (a). 
these particles do not weaken the SCM415/SUS304 bonded interface, perhaps because the particles are too small to cause significant crack initiations. Formation of a chemically-diffused interface with the moderate composition gradient of constituent elements is considered to contribute effectively to the strong bonding at the SCM415/SUS304 interface.

(2) During the annealing at $1000^{\circ} \mathrm{C}$ for $1-2 \mathrm{~min}$, the cold-rolled SCM415/SUS304 interface microstructure composed of severely-deformed grains is fully replaced by the austenite grains due to recrystallization and $\alpha^{\prime}-\gamma$ reverse transformation for the SUS304 and SCM415 layers. These austenite grains have grown continuously to stride across the initial heterointerface, which can be traced by the array of the nanocrystalline silicon-oxide particles those appeared in the gain interiors. This microstructural reconstruction effectively unifies the original interface, and hence it is suggested that the bonding between the dissimilar steels is primary accomplished at this stage.

(3) During the water-quenching from $1000^{\circ} \mathrm{C}$, the martensitic transformation from the SCM415 layer proceeds into the reconstructed austenite grains that have covered the original interface, forming lath martensites that are protruded from the initial interface but terminated within the austenite grain interiors. The termination of the lath growth is reasonably interpreted as a decrease of its mean driving force when growing along the composition gradient; the $\mathrm{Ni} / \mathrm{Cr}$-equivalent values at the lath-terminated regions appear to be consistent with those expected from the Schaeffler diagram. It is indicated that the transformationinduced strain localization is not so significant around the present lath tips, and hence the evolution of the martensite microstructures across the interface does not weaken but efficiently achieves the strong bonding of the austenite/ martensite interface.

\section{Acknowledgement}

This research was supported by LISM (Layer-Integrated Steels and Metals) project funded by Ministry of Education, Culture, Sports, Science and Technology of Japan. The authors gratefully acknowledge stimulating discussions with Prof. Koseki, Prof. Suzuki, Dr. Sugiyama, Dr. Koyama, Dr. Nambu and Dr. Michiuchi.

\section{REFERENCES}

1) F. Carreno, J. Chao, M. Pozuelo and O. A. Ruano: Scr. Mater, 48 (2003), 1135.

2) D. R. Lesuer, C. K. Syn, J. D. Whittenberger and O. D. Sherby: Int. J. Plast., 18 (2002), 155.

3) N. Masahashi, S. Watanabe, S. Hanada, K. Komatsu and G. Kimura: Metall. Mater. Trans. A, 37 (2006), 1665.

4) M. Pozuelo, F. Carreño, C. M. Cepeda-Jiménez and O. A. Ruano: Metall. Mater. Trans. A, 39 (2008), 666.

5) O. D. Sherby, J. Wadsworth, R. D. Caligiuri, L. E. Eiselstein, B. C. Snyder and R. T. Whalen: Scr. Metall., 13 (1979), 941.

6) S. L. Semiatin and H. R. Piehler: Metall. Trans. A, 10A (1979), 97.

7) D. R. Lesuer, C. K. Syn, O. D. Sherby, J. Wadsworth, J. J. Lewandowski and W. H. Hunt, Jr.: Int. Mater. Rev., 41 (1996), 169.

8) H. C. Tsai, J. Wolfenstine and O. D. Sherby: Composites, 22 (1991), 373.

9) D. W. Kum, T. Oyama, O. A. Ruano and O. D. Sherby: Metall. Trans. A, 17A (1986), 1517.

10) B. C. Snyder, J. Wadsworth and O. D. Sherby: Acta Metall., 32 (1984), 919.

11) S. Lee, T. Oyama, J. Wadsworth and O. D. Sherby: Mater. Sci. Eng. A, 154 (1992), 133

12) C. K. Syn, D. R. Lesuer, J. Wolfenstine and O. D. Sherby: Metall. Trans. A, 24A (1993), 1647.

13) S. Nambu, M. Michiuchi, J. Inoue and T. Koseki: Compos. Sci. Technol., 69 (2009), 1936.

14) S. Nambu: Private Communication.

15) M. Michiuchi: Private Communication.

16) I. Gutierrez, J. J. Urcola, J. M. Bilbao and L. M. Villar: Mater. Sci. Technol., 7 (1991), 761.

17) B. Lopez, I. Gutierrez and J. J. Urcola: Mater. Sci. Technol., 12 (1996), 45.

18) R. P. Smith: Acta Metall., 1 (1953), 578.

19) G. E. Murch and C. M. Bruff: Diffusion in Solid Metals and Alloys, Landolt-Börnstein-Group III Condensed Matter, Vol. 26, ed. by H. Bakker et al., Springer-Verlag, Berlin Heidelberg, (1990), 279.

20) J. Pavlovsky, B. Million, K. Ciha and K. Stransky: Mater. Sci. Eng. A, 149 (1991), 105.

21) M. Huang and L. Wang: Metall. Mater. Trans. A, 29 (1998), 3037.

22) A. L. Schaeffler: Met. Prog., 56 (1949), 680.

23) A. Borgenstam, M. Hillert and J. Ågren: Acta Metall. Mater., 43 (1995), 945.

24) B. P. J. Sandvik and C. M. Wayman: Metall. Trans. A, 14A (1983), 809 . 